\title{
28 Research Soure \\ Efficacy and Safety of Combined \\ Phacoemulsification, Intraocular Lens Implantation, \\ Goniosynechialysis, and Trabectome in Patients \\ With Primary Angle-Closure Glaucoma
}

\section{Yu Wang}

Peking University People's Hospital

Zhiqiao Liang

Peking University People's Hospital

\section{Yu Zhang}

Peking University People's Hospital

Hennein Lauren

University of California, San Francisco

\section{Ying Han}

University of California, San Francisco

Huijuan Wu ( $\nabla$ dr_wuhuijuan@126.com )

Peking University People's Hospital

\section{Research Article}

Keywords: phacoemulsification, goniosynechialysis, trabectome, primary angle-closure glaucoma, efficacy and safety

Posted Date: January 18th, 2021

DOl: https://doi.org/10.21203/rs.3.rs-146591/v1

License: (c) (1) This work is licensed under a Creative Commons Attribution 4.0 International License. Read Full License

Version of Record: A version of this preprint was published at Scientific Reports on July 6th, 2021. See the published version at https://doi.org/10.1038/s41598-021-92972-9. 


\section{Abstract}

We evaluated the efficacy and safety of combined phacoemulsification, intraocular lens implantation, goniosynechialysis (GSL), and trabectome in patients with primary angle-closure glaucoma (PACG). In this prospective interventional study, twenty patients (22 eyes) of PACG treated with combined phacoemulsification, intraocular lens implantation, GSL, and trabectome between September 2017 to September 2020 were recruited. The intraocular pressure (IOP), the number of glaucoma medications, and best-corrected visual acuity (BCVA) were recorded at baseline, 1, 3, 6, 12, and 24 months after operation. IOP was decreased significantly from $20.69 \pm 6.90 \mathrm{mmHg}$ at baseline to $15.83 \pm 2.79 \mathrm{mmHg}$ at 24 months' follow-up ( $P=0.043)$. The number of glaucoma medications reduced from $2.76 \pm 1.14$ preoperatively to $0.73 \pm 0.77$ at 24 months' follow-up ( $P=0.026)$. The qualified success rate was $86.8 \%$ at 2 years. The reduction of IOP showed a positive correlation with baseline IOP $(p<0.001)$ and the reduction of the number of glaucoma medications was positively correlated with baseline number of glaucoma medications $(p<0.001)$. There was no vision-threatening complication during and after operation. The combined procedure of phacoemulsification, IOL implantation, GSL, and trabectome was effective and safe for PACG patients. It may provide a new method for PACG patients especially those with long term and extensive goniosynechia.

\section{Introduction}

Primary angle-closure glaucoma (PACG) is a common subtype of glaucoma in China characterized as progressive peripheral anterior synechiae (PAS) that leads to a permanent closure of the anterior chamber angle with elevated intraocular pressure (IOP) and subsequent irreversible optic nerve damage. ${ }^{1}$ Traditional surgeries for PACG include trabeculectomy and glaucoma valve implantation. Potentially dangerous intraoperative and postoperative complications may occur at a frequency that cannot be ignored, including surgery-related bleb scarring, hypotony, bleb leak, and bleb infection, which are associated with a high rate of additional surgeries. ${ }^{2-4}$ Although cataract extraction can deepen a crowded anterior chamber, the adhered angle can still remain. ${ }^{5-7}$ Combined with goniosynechialysis (GSL), phacoemulsification and intraocular lens (IOL) implantation may still fail to achieve a satisfying IOP level resulted from the disabled trabecular meshwork $(T M)^{8-10}$. The success rate was $71.0 \%-92.3 \% 9,11-14$ and subsequent trabeculectomy and glaucoma valve implanation was necessary in $2.9 \%-5 \%$ patients. ${ }^{15-17}$ These limitations promote the development of minimally invasive glaucoma surgery, searching for new, effective and safe therapy.

Trabectome was one of the first-introduced (minimally invasive glaucoma surgery) MIGS and ablates the TM and inner wall of Schlemm's canal (SC) to lower IOP, which is the main part of outflow resistance in the pathophysiology of glaucoma. ${ }^{18,19}$ Considering the need to expose TM tissue during surgery, the trabectome was mainly used in various types of open angle glaucoma (OAG). Previous studies investigated that the effectiveness of trabectome in OAG have demonstrated a $22-35 \%$ decrease in IOP 
and a $18-58 \%$ decrease in the number of IOP-lowering medications during follow-up period between 7 to 90 months. ${ }^{18,20-22}$

Since the main resistance of outflow in PACG is located at the obstructed TM tissue, the normal outflow drainage system of SC provides an opportunity to use trabectome in PACG patients after effective GSL. Although previous study has suggested that the indications of trabectome should be expanded to include narrow angles, ${ }^{23}$ to the best of our knowledge, it has not been used for the treatment of PACG. The purpose of this study is to evaluate the efficacy and safety of combined trabectome with phacoemulsification, IOL implantation, and GSL in PACG patients.

\section{Methods}

\section{Patients}

This study was a prospective study that included 20 patients (22 eyes) diagnosed with PACG and concurrent cataract need surgical intervention to control IOP at the Peking University People's Hospital between September 2017 to September 2020. Among them, 6 were male and 14 were female. All of these 22 eyes received combined phacoemulsification, IOL implantation, GSL, and trabectome. The patients were enrolled in the study when the following baseline criteria were fulfilled: 1 . Synechial angle closure greater than $180^{\circ}$ as demonstrated on gonioscopy and ultrasound biomicroscopy (UBM); 2. Patients with IOP greater than $21 \mathrm{mmHg}$ or patients with normal IOP with the use of at least one anti-glaucoma medication; 3 . The existence of visual disabling cataract. The exclusion criteria were as follows: 1. Patients with a history of prior intraocular surgery (except for laser peripheral iridoctomy, laser iridoplasty, and anterior chamber paracentesis); 2. Patients with prior ocular diseases which affected the anatomy or function of anterior segment, such as history of ocular trauma, uveitis, and proliferative diabetic retinopathy. Written informed consent was obtained for all patients. Our study followed the guidelines of the Declaration of Helsinki and was approved by the Institutional Review Board of Peking University People's Hospital (Beijing, China).

\section{Examination}

Before surgery, all patients underwent a comprehensive ophthalmologic examination, including best corrected visual acuity (BCVA), IOP (Goldmann applanation tonometry), and slit lamp examination. Visual acuity was measured with the Snellen visual chart. Visual field perimetry (Swedish Interactive Threshold Algorithm [SITA] 24 - 2 test of the Humphrey visual field analyzer 750i, Carl Zeiss Meditec, Dublin, CA) was used to evaluate the visual function damage. Gonioscopy and UBM (Aviso, Quantel Medical, Inc., Bozeman, MT, USA) was performed to determine the extent of goniosynechia. The duration between PACG and surgery was also recorded. PAS was defined as a region of irido-trabecular contact that could not be opened by indentation gonioscopy. Follow-up visits were at certain postoperative periods: 1 day, 1 week, 1 month, 3 months, 6 months, 12 months, and 24 months. The subsequent long-term follow-up interval was at 1 year. At every visit, BCVA, IOP, intra-operative or post-operative complications, parameters 
of UBM, and number of IOP-lowering medications were recorded. In this study, the success of surgery was defined as IOP $₫ 21 \mathrm{mmHg}$ with or without IOP-lowering medication.

\section{Surgical procedure: phacoemulsification, IOL implantation, GSL, and trabectome}

All surgeries were performed by the same skilled surgeon (WHJ) who is capable of dealing with both cataract and glaucoma surgeries under topical anesthesia. Standard phacoemulsification was performed through a 2.8-mm superior clear corneal incision and an auxiliary temporal corneal paracentesis. After the implantation of a foldable IOL within the capsular bag, carbachol (1 ml:0.1 mg, Bausch \& Lomb, Shandong, CN) was injected into the anterior chamber to induce miosis. GSL using viscoelastic ( $1 \mathrm{ml}$, Bausch \& Lomb, Shandong, CN) was performed circumferentially. With the assistance of an intraoperative surgical gonioscope, after the anterior segment was refilled with viscoelastic, the surgeon gently pressed against the peripheral iris with a blunt cyclodialysis spatula to exert a backwards pressure on the iris and expose the TM at the nasal side. The temporal clear cornea incision was enlarged to $1.7 \mathrm{~mm}$, through which the trabectome single-use hand piece with the irrigation-aspiration (I/A) system (Neomedix Inc., Tustin, USA) reached the nasal anterior chamber. The TM and inner wall of SC were removed about $90^{\circ}$ to $120^{\circ}$ with a power of $0.8 \mathrm{~W}$. Reflux hemorrhage from SC with the withdrawal of the hand piece was a marker of success. Viscoelastic was removed by using the I/A-system.

All eyes were prescribed pilocarpine 2\% (5ml:25 mg, Bausch \& Lomb Freda, Shandong, CN) three times per day for the first week after surgery, and then two times per day for four more weeks. Tobramycin and dexamethasone eye drops (5 ml, s.a.ALCON-COUVREUR n.v., Rijksweg 14,B-2870 Puurs, Belgium) were prescribed four times per day or more frequently based on postoperative inflammation and intraocular reflux bleeding. The Tobradex were reduced gradually over several weeks after surgery. IOP-lowering medications were prescribed topically based on postoperative IOP.

\section{Statistical analysis}

All the results were analyzed using the software SPSS 19.0 (Chicago, USA). All data was showed as a mean \pm standard deviation. The distribution of the data was validated by the Kolmogorov-Smirnov test. Parametric test (Student's t test) was conducted for some pre- and post-surgical findings. The success of the procedure was analyzed using a Kaplan-Meier analysis. Relevant factors to reduction of IOP and reduction of the number of glaucoma medications were recognized by univariate linear regression, including age, glaucoma duration, baseline IOP, number of glaucoma medications, and the extent of goniosynechia. A p-value of less than 0.05 was considered to be statistically significant.

\section{Results}

Table 1 provides the demographic data of all 20 patients (22 eyes), including 6 males and 14 females. All included patients underwent combined phacoemulsification, IOL implantation, GSL, and trabectome 
surgery. Reflux hemorrhage from SC with the withdrawal of the hand piece was seen in all patients. Mean patient age was 71.68 years,. The mean follow-up time was $12.18 \pm 8.73$ months.

Table 1

Baseline characteristics of all 20 patients ( 22 eyes)

\begin{tabular}{|ll|}
\hline Male-female ratio & $6: 14$ \\
\hline Mean age (years) & $71.68 \pm 6.28$ (range, 63-85) \\
\hline Mean glaucoma duration (months) & $53.35 \pm 58.61$ (range, 3-192) \\
\hline Mean follow-up period (months) & $12.18 \pm 8.73$ (range, 1-24) \\
\hline Mean preoperative BCVA & $0.38 \pm 0.24$ \\
\hline Mean preoperative IOP (mmHg) & $21.07 \pm 6.56$ \\
\hline Mean number of preoperative IOP-lowering drugs & $2.64 \pm 1.23$ (range, 0-4) \\
\hline Mean extent of goniosynechia (degrees) & $319.09 \pm 64.94$ (range, 180-360) \\
\hline Visual field defect (MD) & $-19.14 \pm 9.07$ (range, -1.02 -30.26) \\
\hline BCVA = best corrected visual acuity; IOP = intraocular pressure; MD = mean deviation \\
\hline
\end{tabular}

The mean IOP at baseline and postoperatively is demonstrated in Fig. 1. The mean preoperative IOP in patients with PACG was $20.69 \pm 6.90 \mathrm{mmHg}$, which decreased to $15.83 \pm 2.79(-23 \%)$ at the mean followup period of 12.18 months. Before surgery, patients with PACG used $2.68 \pm 1.17$ IOP-lowering medications which decreased to $1.17 \pm 0.98(-56 \%)$ at 12.18 months' follow-up (Table 2, Fig. 1).

Table 2

IOP and number of IOP-lowering medications before and after surgery at each follow-up

\begin{tabular}{|lllll|}
\hline & IOP $(\mathrm{mmHg})$ & $\boldsymbol{p}$ & Number of medications & $\boldsymbol{p}$ \\
\hline Baseline $(\mathrm{n}=22)$ & $20.69 \pm 6.90$ & & $2.68 \pm 1.17$ & \\
\hline 1 month $(\mathrm{n}=22)$ & $13.33 \pm 3.99$ & $<0.01$ & $0.32 \pm 0.57$ & $<0.01$ \\
\hline 3 months $(\mathrm{n}=18)$ & $13.19 \pm 3.54$ & $<0.01$ & $0.44 \pm 0.71$ & $<0.01$ \\
\hline 6 months $(\mathrm{n}=14)$ & $14.75 \pm 3.78$ & $<0.01$ & $0.64 \pm 0.75$ & $<0.01$ \\
\hline 12 months $(\mathrm{n}=12)$ & $15.50 \pm 3.06$ & 0.022 & $1.00 \pm 0.74$ & 0.015 \\
\hline 24 months $(\mathrm{n}=6)$ & $15.83 \pm 2.79$ & 0.043 & $1.17 \pm 0.98$ & 0.026 \\
\hline IOP $=$ intraocular pressure & & & \\
\hline
\end{tabular}

For all patients, the postoperative BCVA was significantly improved compared to baseline (Table 3), with the exception of two eyes whose VA were 0.05 (MD: -30.26) and light perception respectively with a 
severe optic nerve damage.

Table 3

Best corrected visual acuity before surgery and 1 month after surgery

\begin{tabular}{|llllllll|}
\hline Number & & & & & & \\
\hline & $0 \sim 0.1$ & $0.1 \sim<0.3$ & $0.3 \sim<0.5$ & $\geq 0.5$ & Mean \pm SD & $p$ \\
\hline Baseline & 4 & 5 & 3 & 10 & $0.39 \pm 0.23$ & \\
\hline 1 month postoperatively & 2 & 4 & 2 & 14 & $0.58 \pm 0.33$ & 0.03 \\
\hline SD = standard deviation & & & & & & \\
\hline
\end{tabular}

Gonioscopy and UBM revealed that the anterior chamber angle of all 22 eyes was closed to different extent at baseline. After operation, the condition was significantly improved and the position of ablation could be clearly seen at each follow-up. Goniosynechia in patients before and after operation is listed in Table 4. A representative case is shown in Fig. 2.

Table 4

Goniosynechia in patients before and after operation.

\begin{tabular}{|llllll|}
\hline Goniosynechia & Open angle & $\begin{array}{l}\mathbf{5 9 0} \\
\text { Degree }\end{array}$ & $\mathbf{> 9 0} \leq 180$ Degree & $>180 \leq 270$ Degree & $>\mathbf{2 7 0}$ \\
\hline Baseline & 0 & 0 & 3 & 4 & Degree \\
\hline 1 month & 4 & 5 & 3 & 2 & 15 \\
\hline 3 months & 3 & 6 & 2 & 0 & 0 \\
\hline 6 months & 4 & 4 & 2 & 0 & 0 \\
\hline 12 months & 4 & 2 & 1 & 0 & 0 \\
\hline 24 months & 2 & 0 & 1 & 0 & 0 \\
\hline
\end{tabular}

Relevant factors to reduction of IOP and reduction of the number of glaucoma medications were recognized by univariate linear regression. As demonstrated by the univariate analysis, the reduction of IOP showed a positive correlation with baseline IOP $(p<0.001)$ and the reduction of the number of glaucoma medications was positively correlated with baseline number of glaucoma medications $(p<$ 0.001). (Table 5) 
Table 5

Univariate regression analysis for factors correlated with the reduction of IOP $\left(R^{2}=0.649\right)$ and reduction of the number of glaucoma medications $\left(R^{2}=0.832\right)$.

\begin{tabular}{|c|c|c|c|c|}
\hline & \multicolumn{2}{|c|}{ Reduction of IOP } & \multicolumn{2}{|c|}{$\begin{array}{l}\text { Reduction of glaucoma } \\
\text { medications }\end{array}$} \\
\hline & $\begin{array}{l}\text { Univariate } \\
\text { coefficient }\end{array}$ & $p$ & $\begin{array}{l}\text { Univariate } \\
\text { coefficient }\end{array}$ & $p$ \\
\hline Age & 0.127 & 0.610 & -0.041 & 0.448 \\
\hline Glaucoma duration & -0.022 & 0.386 & 0.001 & 0.888 \\
\hline Baseline IOP & 0.840 & $<.001$ & -0.044 & 0.385 \\
\hline $\begin{array}{l}\text { Baseline number of glaucoma } \\
\text { medications }\end{array}$ & 1.612 & 0.220 & 1.136 & $<0.001$ \\
\hline Goniosynechia before operation & 2.480 & 0.234 & 0.222 & 0.634 \\
\hline
\end{tabular}

Kaplan-Meier analysis showed a qualified success rate of $86.8 \%$ for PACG at 2 years. No complications occurred during the surgery. All eyes presented with anterior chamber hemorrhage due to reflux hemorrhage from SC, and was absorbed withour additional treatment. Among the 22 eyes, re-occurrence of PAS at the position of the removed TM tissue was observed in only two eyes, which received peripheral argon laser iridoplasty as soon as the reformation of PAS observed at the 1 month visit. No other postoperation complications occurred.

\section{Discussion}

In the current study, we explored the efficacy and safety of trabectome combined with phacoemulsification, IOL implantation, and GSL in PACG patients. The results demonstrate that with the assistance of phacoemulsification and re-establishing the anterior chamber angle, trabectome showed an effective IOP-lowering effect with satisfied reduction of the number of IOP-lowering medications at 2 years.

PACG is a kind of ocular disorder characterized by anterior chamber angle closure, which is the main cause of blindness worldwide as well as in China. The main pathophysiological mechanisms of angle closure are pupillary block and iris plateau configuration. The purpose of treatment is to open the outflow drainage passage and reduce IOP through medical and/or surgical treatment. Traditional surgeries for PACG, including trabeculectomy, glaucoma valve implantation, and phacotrabeculectomy, has traditionally been used to treat a wide range of PAS patients, complications of which include shallow anterior chamber, cataract progression, filtering bleb scar, congenital arthritis, etc. Phacoemulsification with or without GSL and has been shown in many studies to lower IOP in PACG. ${ }^{17,24}$ Although 
phacoemulsification with GSL shows great advantages over traditional surgeries from the point of view of complications, IOP-lowering effect was not always satisfied, especially for patients with long duration of angle-closure glaucoma. ${ }^{8,9,25}$ Tian et al. ${ }^{25}$ compared the efficacy of phacoemulsification combined with GSL in the treatment of acute and chronic angle closure patients. The success rate in the chronic group (acute vs chronic: $100 \%$ vs $64.3 \%$ ) was lower. Compared the efficacy of phacoemulsification combined with GSL and phacoemulsification alone in patients with well controlled chronic angle closure glaucoma and cataract.Lee et al. ${ }^{8}$ compare the effect of phacoemulsification combined with GSL and phacoemulsification alone in patients with medically well- controlled chronic angle-closure glaucoma with cataracts. They found there was no significant difference between two groups in lowering IOP and suggested that it is difficult to attain more benefits from additional GSL. Husain et al. ${ }^{9}$ concluded similar results with Lee et al. through a randomized clinical trial. Furthermore, Wang et al. ${ }^{10}$ reviewed and analyzed seven randomized controlled trials to determine the effect of phacoemulsification and GSL compared to phacoemulsification alone in ACG patients. A "low to moderate quality evidence" was disclosed that phacoemulsification and GSL surgery lead to an equivalent IOP-lowering effect compared to phacoemulsification alone. This indicates that the temporary re-opening of the angle is insufficient in chronic PACG. Even if the angle is kept open for a longer period of time, the re-exposed TM might fail to function. These findings had led to an ongoing effort to explore new strategies that avoid these pitfalls and offer a longer lasting result.

In our study, with the assistance of phacoemulsification and GSL, trabectome offers an effective IOPlowering effect with satisfied reduction of the number of IOP-lowering medications for PACG patients at 2 years. The mean glaucoma duration was $53.35 \pm 58.61$ months and the mean extent of goniosynechia before operation was $319.09 \pm 64.94$ degrees in our study. Seventeen eyes had been suffering from it for more than 1 year, and 15 of 22 eyes presented the 360 degrees of synechial angle closure. For this group of patients with long term and extensive goniosynechia, we not only performed phacoemulsification and GSL, but trabectome, which creates a cleft that connects the anterior chamber to the SC, allowing aqueous to enter the collector channels more directly without the resistance of the TM. Previous studies $^{26,27}$ have proved that re-establishing a normal open angle configuration with goniosynechiolysis is not sufficient for successful IOP-lowering. Shihota et al. ${ }^{28}$ proposed that PAS and angle closure causes pigment accumulation and non-inflammatory degeneration of the TM. For patients with PAS less than 6 months to 1 year, TM function could be preserved and the effect of goniosynechiolysis might be satisfied. ${ }^{29,30}$ We suggest that the TM function of PACG patients with long term and extensive goniosynechia have undergone irreversible damage. White et al. ${ }^{31}$, Zhao et al. ${ }^{11}$ and Husian et al. ${ }^{9}$ hold the similar opinion with us, which give reasonable explanation that IOP-lowering effect was not always satisfied for patients with long duration of angle-closure glaucoma.

Another potential limitation of goniosynechiolysis combined phacoemulsification or not is that the lowering of the IOP may not be satisfied due to reformation of PAS. Lee et al. ${ }^{8}$ reported that postoperative PAS reformation was observed in 5 patients (33.3\%) after goniosynechiolysis. The recurrence of PAS was more serious than that before operation and the main site was identical before operation. The rate of PAS 
recurrence was 30\%-100\% after goniosynechiolysis, and the rate was much higher with long duration of

PAS. ${ }^{8,16,25}$ However, there was only 2 eyes (9\%) experienced PAS recurrence in current study. We speculated that trabecutome combined with goniosynechiolysis makes the anterior chamber angle wider and the space between the peripheral iris and SC larger, which may be another obvious advantage of trabecutome. Even if there is obvious inflammatory reaction after goniosynechiolysis, PAS is hard to reformation.

Relevant factors to the reduction in IOP and the number of medications were determined by regression analysis. As showed by the univariate analysis, we found greater reduction in IOP and the number of medications in PACG patients presented with higher baseline IOP and more IOP-lowering medications respectively. We hypothesized that eyes with higher IOP and more medications may have better results because they will undergo early surgery after diagnosis.

The following study limitations should be considered. First, the sample size was small and the follow-up period was short. Larger-scale, longer-follow up trials would provide stronger evidence to compare the efficacy of this procedure with that of other operations. Second, we only enrolled PACG patients with concurrent cataract who received phacoemulsification, IOL implantation, GSL, and trabectome without control group. Additional studies with two groups who receive phacoemulsification, intraocular IOL and GSL with or without trabectome may be more helpful to elucidate the efficacy and safety of combined trabectome.

In conclusion, the combined procedure of phacoemulsification, IOL implantation, GSL, and trabectome was a possibly effective and safe surgical choice for lowering the IOP and reducing the number of antiglaucoma medications in PACG patients. It may provide a brand-new method for PACG patients especially those with long term and extensive goniosynechia. Further longitudinal studies with larger sample size, control group, and subgroup analysis are necessary to demonstrate the advantage of this procedure.

\section{Declarations}

\section{Data availability}

Datasets from the current study are not publicly available due to compliance to privacy. Summary statistics are available from the corresponding author on reasonable request.

\section{Author contribution}

This work was supported by the program of development and cultivation of medical innovative varieties and industrial support, Beijing Municipal Science and Technology Commission [grant number Z191100007619045]; and National Natural Science Foundation of China [grant number 61634006]. The funders had no role in the study design, data collection and analysis, decision to publish or preparation of the manuscript. Y.W., Z.Q.L and H.J.W designed the study. Y.W. and Z.Q.L collected data and performed 
the statistical analysis. Y.Z. and H.J.W interpreted the results. Y.W. and Z.Q.L. wrote the manuscript. L.H. and Y.H. provided English editing. All authors commented on and approved the manuscript. Y.W. and Z.Q.L contributed equally to this study, and should be considered as co-first authors.

\section{Competing interests}

The authors declare no competing interests.

\section{Additional information}

Correspondence and requests for materials should be addressed to H.J.W.

\section{References}

1. Sun, X. et al. Primary angle closure glaucoma: What we know and what we don't know. Progress in retinal and eye research. 57, 26-45 https://doi.org/10.1016/j.preteyeres.2016.12.003 (2017).

2. Bellucci, R., Perfetti, S., Babighian, S., Morselli, S. \& Bonomi, L. Filtration and complications after trabeculectomy and after phaco-trabeculectomy. Acta ophthalmologica Scandinavica. Supplement. 44-45 https://doi.org/10.1111/j.1600-0420.1997.tb00473.x (1997).

3. Ohtomo, K., Mayama, C., Ueta, T. \& Nagahara, M. Outcomes of Late-Onset Bleb-Related Endophthalmitis Treated with Pars Plana Vitrectomy. Journal of ophthalmology 2015, 923857, doi:10.1155/2015/923857 (2015).

4. Jongsareejit, B. et al. Efficacy and complications after trabeculectomy with mitomycin $\mathrm{C}$ in normaltension glaucoma. Japanese journal of ophthalmology. 49, 223-227 https://doi.org/10.1007/s10384-004-0181-9 (2005).

5. Lai, J. S., Tham, C. C. \& Lam, D. S. The efficacy and safety of combined phacoemulsification, intraocular lens implantation, and limited goniosynechialysis, followed by diode laser peripheral iridoplasty, in the treatment of cataract and chronic angle-closure glaucoma. Journal of glaucoma. 10, 309-315 https://doi.org/10.1097/00061198-200108000-00011 (2001).

6. Teekhasaenee, C. \& Ritch, R. Combined phacoemulsification and goniosynechialysis for uncontrolled chronic angle-closure glaucoma after acute angle-closure glaucoma. Ophthalmology. 106, 669-674 discussion 674 - 665 https://doi.org/10.1016/s0161-6420(99)90149-5 (1999).

7. Sengupta, S. et al. Intraocular Pressure Reduction after Phacoemulsification versus Manual SmallIncision Cataract Surgery: A Randomized Controlled Trial. Ophthalmology. 123, 1695-1703 https://doi.org/10.1016/j.ophtha.2016.04.014 (2016).

8. Lee, C. K. et al. Effect of Goniosynechialysis During Phacoemulsification on IOP in Patients With Medically Well-controlled Chronic Angle-Closure Glaucoma. Journal of glaucoma. 24, 405-409 https://doi.org/10.1097/ijg.0000000000000043 (2015).

9. Husain, R. et al. Efficacy of Phacoemulsification Alone vs Phacoemulsification With Goniosynechialysis in Patients With Primary Angle-Closure Disease: A Randomized Clinical Trial. 
JAMA ophthalmology. 137, 1107-1113 https://doi.org/10.1001/jamaophthalmol.2019.2493 (2019).

10. Wang, N. \& Jia, S. B. Phacoemulsification with or without goniosynechialysis for angle-closure glaucoma: a global Meta-analysis based on randomized controlled trials. International journal of ophthalmology. 12, 826-833 https://doi.org/10.18240/ijo.2019.05.20 (2019).

11. Zhao, X. J., Yang, X. X., Fan, Y. P., Li, B. H. \& Li, Q. Comparison of Combined Phacoemulsification, Intraocular Lens Implantation, and Goniosynechialysis With Phacotrabeculectomy in the Treatment of Primary Angle-Closure Glaucoma and Cataract. Asia-Pacific journal of ophthalmology (Philadelphia, Pa.). 2, 286-290 https://doi.org/10.1097/APO.0b013e318299df62 (2013).

12. Kameda, T., Inoue, T., Inatani, M. \& Tanihara, H. Long-term efficacy of goniosynechialysis combined with phacoemulsification for primary angle closure. Graefes Arch Clin Exp Ophthalmol. 251, 825$830 \mathrm{https}: / /$ doi.org/10.1007/s00417-012-2091-8 (2013).

13. Rodrigues, I. A. et al. Aqueous outflow facility after phacoemulsification with or without goniosynechialysis in primary angle closure: a randomised controlled study. The British journal of ophthalmology. 101, 879-885 https://doi.org/10.1136/bjophthalmol-2016-309556 (2017).

14. Fakhraie, G. et al. Phacoemulsification and goniosynechialysis for the management of refractory acute angle closure. European journal of ophthalmology. 22, 714-718 https://doi.org/10.5301/ejo.5000101 (2012).

15. Harasymowycz, P. J. et al. Phacoemulsification and goniosynechialysis in the management of unresponsive primary angle closure. Journal of glaucoma. 14, 186-189 https://doi.org/10.1097/01.ijg.0000159131.38828.85 (2005).

16. Nie, L. et al. Combined Phacoemulsification and Goniosynechialysis under an Endoscope for Chronic Primary Angle-Closure Glaucoma. Journal of ophthalmology 2018, 8160184, doi:10.1155/2018/8160184 (2018).

17. Zhang, H., Tang, G. \& Liu, J. Effects of Phacoemulsification Combined With Goniosynechialysis on Primary Angle-closure Glaucoma. Journal of glaucoma. 25, e499-503 https://doi.org/10.1097/ijg.0000000000000297 (2016).

18. Jordan, J. F. et al. Trabectome surgery for primary and secondary open angle glaucomas. Graefes Arch Clin Exp Ophthalmol. 251, 2753-2760 https://doi.org/10.1007/s00417-013-2500-7 (2013).

19. Bendel, R. E. \& Patterson, M. T. Long-term Effectiveness of Trabectome (Ab-interno Trabeculectomy) Surgery. J Curr Glaucoma Pract. 12, 119-124 https://doi.org/10.5005/jp-journals-10028-1256 (2018).

20. Mosaed, S. The First Decade of Global Trabectome Outcomes. European Ophthalmic Review. 08, 113 https://doi.org/10.17925/EOR.2014.08.02.113 (2014).

21. Ting, J., Damji, K. \& Stiles, M. Ab interno trabeculectomy: Outcomes in exfoliation versus primary open-angle glaucoma. Journal of cataract and refractive surgery. 38, 315-323 https://doi.org/10.1016/j.jcrs.2011.08.043 (2012).

22. Minckler, D., Mosaed, S., Francis, B., Loewen, N. \& Weinreb, R. Clinical Results of Ab Interno Trabeculotomy Using the Trabectome for Open-Angle Glaucoma: The Mayo Clinic Series in 
Rochester, Minnesota. American journal of ophthalmology. 157, 1325-1326

https://doi.org/10.1016/j.ajo.2014.02.030 (2014).

23. Bussel, I. I., Kaplowitz, K., Schuman, J. S. \& Loewen, N. A. Outcomes of ab interno trabeculectomy with the trabectome by degree of angle opening. The British journal of ophthalmology. 99, 914-919 https://doi.org/10.1136/bjophthalmol-2014-305577 (2015).

24. Imaizumi, M., Takaki, Y. \& Yamashita, H. Phacoemulsification and intraocular lens implantation for acute angle closure not treated or previously treated by laser iridotomy. Journal of cataract and refractive surgery. 32, 85-90 https://doi.org/10.1016/j.jcrs.2005.11.014 (2006).

25. Tian, T., Li, M., Pan, Y., Cai, Y. \& Fang, Y. The effect of phacoemulsification plus goniosynechialysis in acute and chronic angle closure patients with extensive goniosynechiae. BMC ophthalmology. 19, 65 https://doi.org/10.1186/s12886-019-1070-9 (2019).

26. Johnson, M., Shapiro, A., Ethier, C. R. \& Kamm, R. D. Modulation of outflow resistance by the pores of the inner wall endothelium. Investigative ophthalmology \& visual science. 33, 1670-1675 (1992).

27. Tanihara, H., Nishiwaki, K. \& Nagata, M. Surgical results and complications of goniosynechialysis. Graefes Arch Clin Exp Ophthalmol. 230, 309-313 https://doi.org/10.1007/bf00165936 (1992).

28. Sihota, R. et al. The trabecular meshwork in acute and chronic angle closure glaucoma. Indian journal of ophthalmology. 49, 255-259 (2001).

29. Shingleton, B. J., Chang, M. A., Bellows, A. R. \& Thomas, J. V. Surgical goniosynechialysis for angleclosure glaucoma. Ophthalmology. 97, 551-556 https://doi.org/10.1016/s0161-6420(90)32542-3 (1990).

30. Campbell, D. G. \& Vela, A. Modern goniosynechialysis for the treatment of synechial angle-closure glaucoma. Ophthalmology. 91, 1052-1060 https://doi.org/10.1016/s0161-6420(84)34195-1 (1984).

31. White, A. J., Orros, J. M. \& Healey, P. R. Outcomes of combined lens extraction and goniosynechialysis in angle closure. Clinical \& experimental ophthalmology. 41, 746-752 https://doi.org/10.1111/ceo.12121 (2013).

\section{Figures}




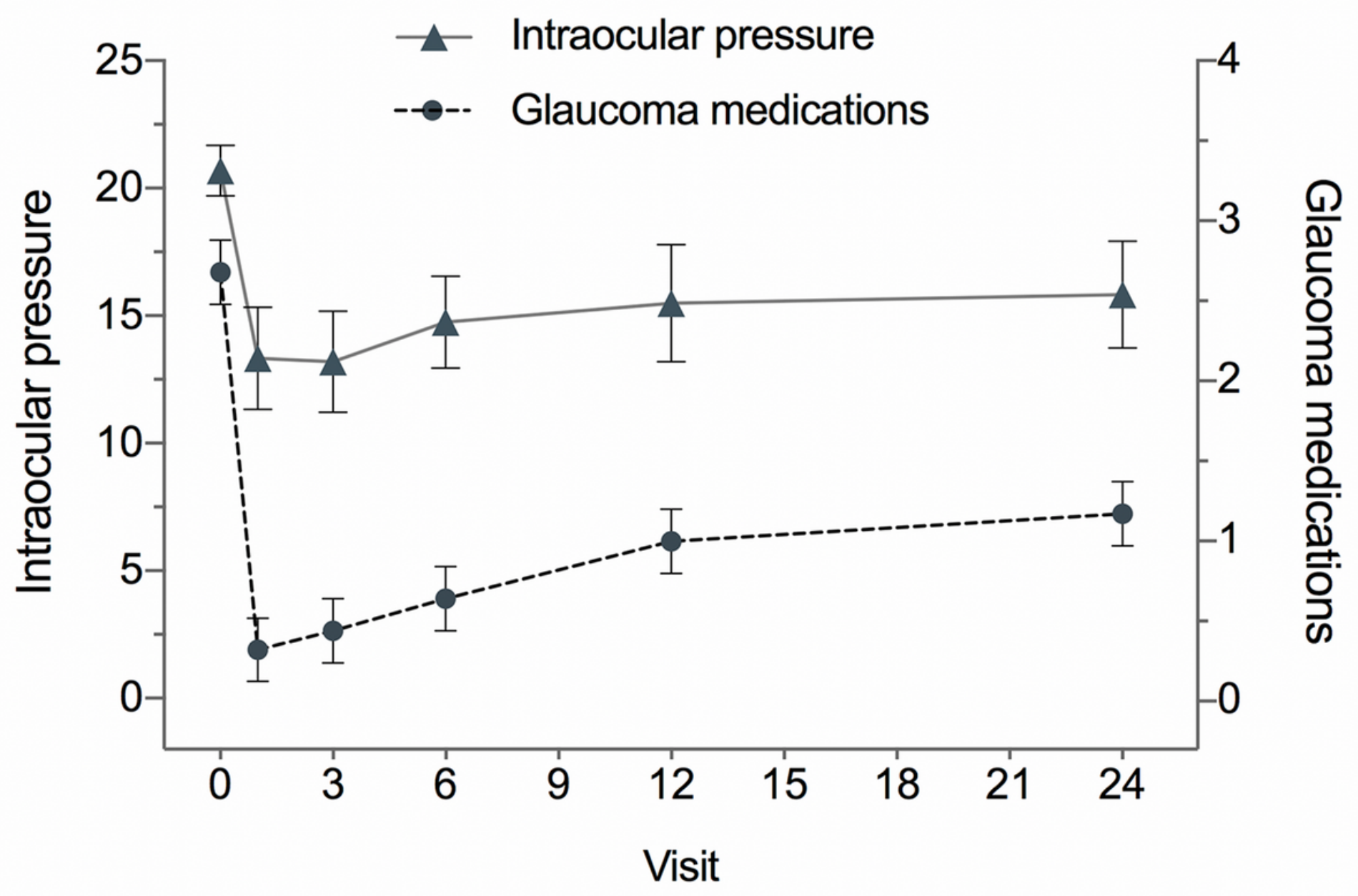

Figure 1

Serial changes of intraocular pressure (IOP) and glaucoma medications from baseline at each follow-up visit. The IOP was significantly decreased at each follow-up visit (1 month: $p<0.01 ; 3$ months: $p<0.01 ; 6$ months: $p<0.01 ; 12$ months: $p=0.022 ; 24$ months: $p=0.043$, respectively). The IOP-lowering medications was significantly decreased at each follow-up visit (1 month: $p<0.01 ; 3$ months: $p<0.01 ; 6$ months: $p<0.01 ; 12$ months: $p=0.015 ; 24$ months: $p=0.026$, respectively). 


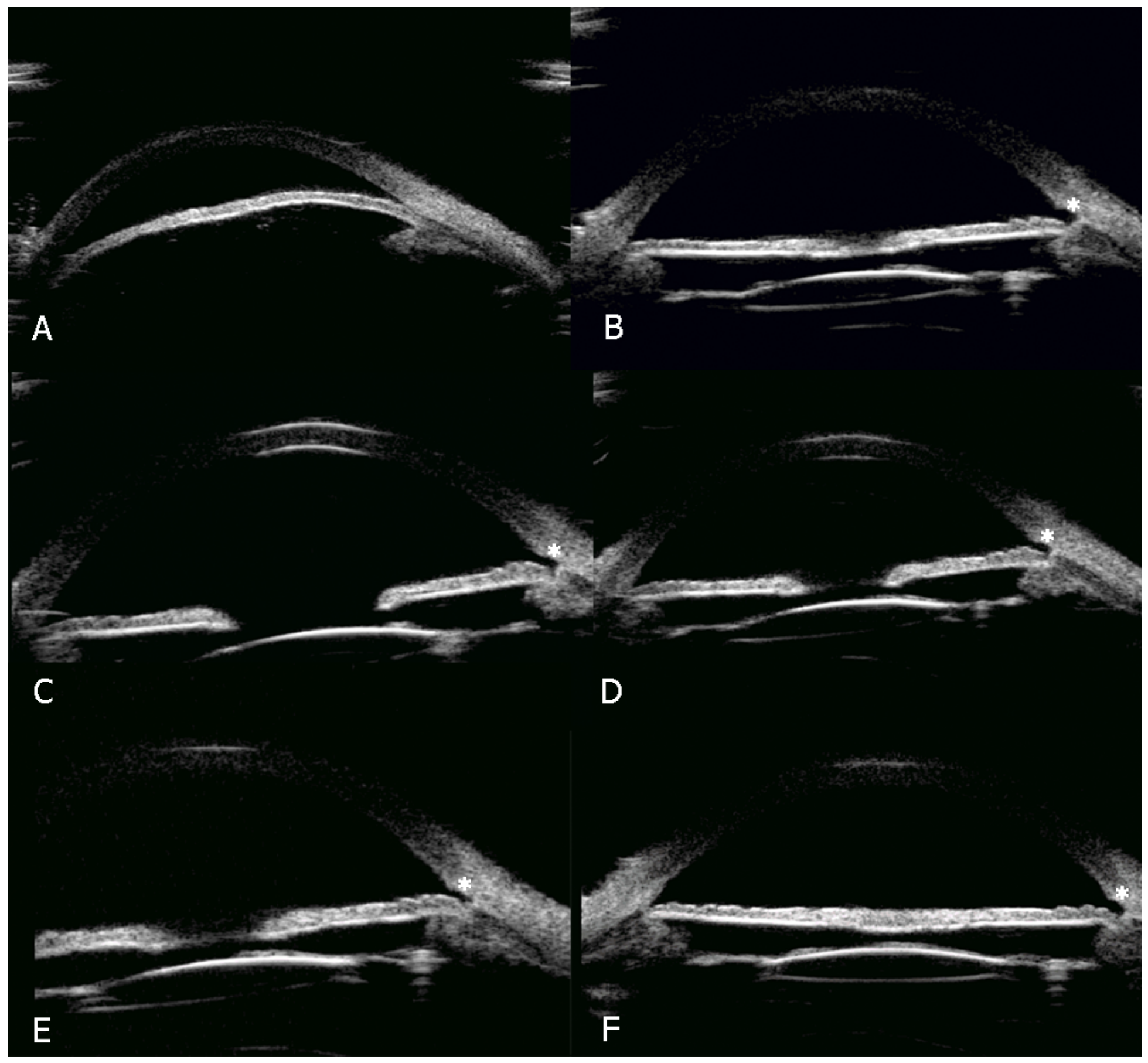

\section{Figure 2}

Series ultrasound biomicroscopy (UBM) image of a representative case of primary angle-closure glaucoma after combined phacoemulsification, intraocular lens implantation, goniosynechialysis and trabectome. An 81-year-old gent with blurred vision for 10 years in the left eye. His best correct visual acuity (BCVA) was 0.05 and intraocular pressure (IOP) was $20.5 \mathrm{mmHg}$ with the use 3 anti-glaucoma medications at baseline. A. UBM image before operation showed that the anterior chamber angle on nasal side was closed. B. UBM image 1 month, 3 months, 6 months, 12 months, and 24 months after operation presented the narrow chamber angle had been obviously widened. At last visit, IOP dropped to $14 \mathrm{mmHg}$ with only 1 medication. White asterisks represent the position that undergone trabectome. 